\title{
OPTICAL POLARIZATION of M 82
}

\author{
N.Neininger ${ }^{1}$, R. Beck ${ }^{1}$, F. Backes ${ }^{2} \quad(*)$ \\ Max-Planck-Institut für Radioastronomie, Auf dem Hügel 69 \\ D-5300 Bonn 1, Fed. Rep. of Germany \\ Landessternwarte Heidelberg, Königstuhl \\ D-6900 Heidelberg 1, Fed. Rep. of Germany
}

\begin{abstract}
We observed a sample of nearby galaxies using the CCD-Polarimeter of the Landessternwarte Heidelberg in the R-Band with the 1.23-m telescope of the MPIA at Calar Alto. In most cases polarization was found but the polarization structures cannot be easily related to a single phenomenon. If there is a strong point source, Rayleigh scattering leads to circular patterns of the polarization vectors. If dust grains are aligned in a magnetic field, this so-called Davis-Greenstein-mechanism gives more uniformly distributed E-vectors, which can be distinguished from the Rayleigh scattering. The example of M 82 given here shows both kinds of polarized light. There is evidence of a strong magnetic field component perpendicular to the disk which seems to have its origin in the center of the galaxy. This is consistent with theoretical models as well as with previous observations.
\end{abstract}

\section{Data aquisition and reduction}

From each object 8 pictures were made with a sheet polarizer in front of the CCD which was rotated in steps of 45 degrees. The exposure time was 5 min each for the example given here. The pictures were cleaned and divided by a flatfield in the usual way, and a fitted background was subtracted. Then the flux of the stars was measured and the frames were calibrated with these values to eliminate atmospheric variations and foreground polarization in the Galaxy. On the resulting pictures a discrete Fourier analysis was performed for each pixel. We get one picture representing $Q$, another for $U$ and a third one which contains the total intensity. These were used to compute the polarized intensity, the degree of polarization and the angle of the polarization vectors.

\section{Results}

The picture of the polarized intensity of M 82 (Fig. 1) shows more small-scale features than the picture of the total intensity. This may be due to the fact that the polarized light has its main origin in the outer layers of dust and gas, whereas the total intensity adds all light sources along the line of sight. There seem to be at least two different patterns: the widespread distribution of Rayleigh-scattered

\footnotetext{
* Visiting Astronomers, German-Spanish Astronomical Center, Calar Alto, operated by the Max-Planck-Institut fur Astronomie Heidelberg jointly with the Spanish National Commission for Astronomy.
} 


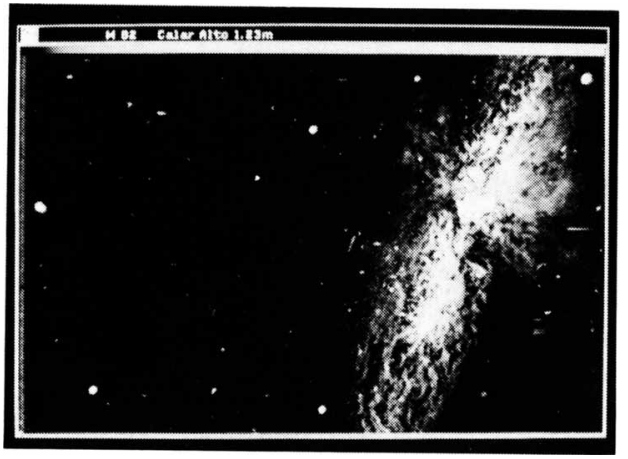

Fig. 1 : Polarized Intensity of M 82

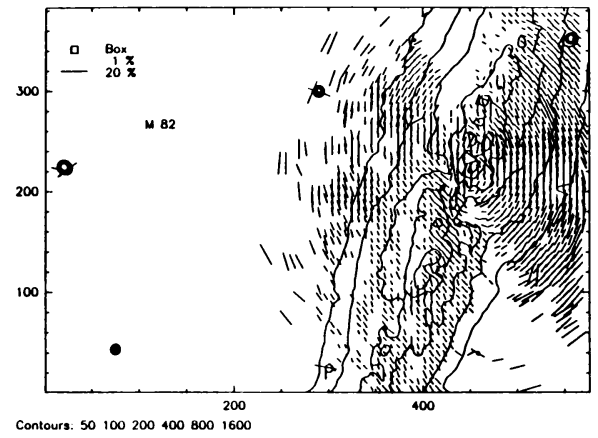

Fig. 2 : Polarization vectors of $\mathrm{M} 82$

light in the the halo of M 82 and the filamentary appearance of large-scale magnetic fields in the disk. Of course, the halo is by far fainter, so the polarized intensity is weaker than in the disk, although the degree of polarization is higher.

The plot of the polarization vectors (Fig. 2) is clearly dominated by circular structures from Rayleigh scattered light out of the very bright nucleus. The polarization is stronger at the northern side (to the right), as is known from other papers ([1], for example). Seen from the halo, the nucleus must be brighter than the disk to produce this pattern. In addition there must be quite a large amount of dust and gas in the halo to give such an amount of scattered light. This has been investigated by several authors (see, for example [1], [2], [3]).

But, as can be seen in polarized intensity, there must be some other contributions to the polarization in the disk that lead to the filaments in Fig. 1 and to the alignment of the vectors in the $z$ direction out of the nucleus, just in the dust band from SE to NW, that makes the waist in the contours. The filaments should belong to a magnetic field component aligned with the disk of this galaxy, whereas the structures near the center correspond to a recently found bipolar outflow out of the nucleus of $\mathrm{M} 82$ [4] and can be explained by theories that use a ring current around the center to induce a poloidal magnetic field [5]. This ring current could exist in the molecular ring which has been found in $\mathrm{CO}$-measurements and flows around the nucleus of $\mathrm{M} 82[6]$.

\section{References:}

[1 ] Bingham,R.G.;McMullan,D.; Pallister,W.S. ; White,C.; Axon, D.J.; Scarrott,S.M.: Nature 259, 463-465 (1976)

[2] Notni, P. : Astron. Nachr. 306, 273-281 (1985)

[3] Solinger, A.B. ; Markert, T. : Astrophys. Journal 197, 309-315 (1975)

[4] Bland,J ; Tully, R.J.: Nature 334, 43-45 (1988)

[5] Lesch, H. ; Crusius, A. ; Schlickeiser, R. ; Wielebinski, R. : Astron. Astrophys 217, 99-107 (1989) and Lesch et al. in this proceeding

[6] Loiseau,N.; Nakai,N.; Sofue,Y.; Wielebinski, R.; Reuter, H.-P.; Klein, U.: Astron. Astrophys. (in press) (1989) 